\title{
AC IMPEDANCE OF ELECTRO-ACOUSTICALLY ACTIVE SEMICONDUCTORS
}

\author{
W. WESTERA, R.J.J. ZIJLSTRA and M.A. van DIJK \\ Fysisch Laboratorium, Rijksuniversiteit Utrecht, The Netherlands
}

Received 1 April 1980

\begin{abstract}
The ac small-signal impedance of electro-acoustically active semiconductors is calculated. Essential for the ac description presented is the introduction of potential troughs that are coupled to the amplified acoustic waves. The calculated impedance shows a frequency dependent behaviour and resonances which are in good agreement with experimental results for CdS.
\end{abstract}

1. Introduction. For many years now continuous interest has been shown in the electro-acoustic effect, i.e. the amplification of travelling acoustic waves through interaction with supersonic drifting charge carriers in piezo-electric semiconductors.

As a first step White [1] gave in 1962 a linear description of the electro-acoustic effect known as the linear small-signal gain theory. In 1965 Greebe [2] used the linear small-signal gain theory to calculate the consequences that boundary conditions have on the impedance of semiconducting piezo-electric plates. Strong nonlinear interaction of space charge waves with stress waves, however, restricts the usefulness of linear theories.

The most prominent nonlinear effects appearing in the sound amplification process are current saturation, electro-acoustic domain formation, current oscillations, parametric interaction of sound waves, large current fluctuations in the saturation current, and impedance effects. Several authors [3-7] have tried to describe these nonlinear phenomena. Since, however, descriptions of this kind, which start from basic principles, are very difficult, many of these phenomena are not yet understood quantitatively.

In 1967 Moore [6] avoided the use of nonlinear equations by suggesting that the observed current saturation and current fluctuations are caused by bunching of free charge carriers in potential troughs which are coupled to the amplified acoustic waves via the piezo-electric effect. He described the observed current fluctuations in CdS by a trough creation-annihilation process.

In $1978 \mathrm{Zijlstra}$ and Gielen [7] modified this theory by accounting for transit time effects in a local description. They neglected the displacement and the diffusion current in the expression for the current density and assumed that the creation and annihilation of troughs are independent of electric field strength. As a consequence they found a frequency-independent impedance. Experimentally, however, the observed ac impedance of electroacoustically active CdS crystals turned out to be frequency dependent. In fact experimental results showed in addition to the familiar dielectric roll-off, another, lower frequency roll-off in the range of $1 \mathrm{MHz}$.

The aim of this paper is to show that the observed frequency dependence of the ac impedance can be explained if the local description is extended by taking into account diffusion, space charge, displacement current and the electric field dependence of the trough creation and annihilation rates. It should be noted that this calculation holds for crystals where a continuous type of amplified acoustic flux is observed (i.e. where no electro-acoustic domains are observed) and when a one-dimensional description obtains.

2. Theory. We consider here an $n$-type piezo-electric semiconducting crystal, where the electric field is applied along a symmetry axis, the $x$-axis. In the case of CdS the $x$-axis coincides with the $c_{6}$-axis.

Current saturation is explained by the assumption that part of the electrons in the conduction band are trapped 
in potential troughs which are associated with the large amplitude acoustic waves and which therefore move with the sound velocity. If we assume that these troughs are spontaneously created and annihilated throughout the crystal, the master equation for the trough density $n_{\mathrm{t}}$ reads [7]

$\partial n_{\mathrm{t}} / \partial t=p-b-v_{\mathrm{s}} \partial n_{\mathrm{t}} / \partial x$

where $p$ is the creation rate of troughs per unit volume, $b$ the annihilation rate of troughs per unit volume, $t$ is time and $v_{\mathrm{s}}$ the trough velocity.

Although the inclusion of diffusion and space charge is important in the ac case it can be shown that for the steady state these can be neglected ${ }^{\ddagger 1}$. This implies that the steady state values of the electric field strength $\bar{E}$, the free electron density $\bar{n}_{\mathrm{d}}$ and the density of electrons trapped by troughs $\bar{n}_{\mathrm{s}}$ are independent of $x$ [7]. In addition we make the approximation that each trough contains $N$ electrons, where $N$ is not explicitly dependent on $x$. The density of trapped carriers is given by

$n_{\mathrm{s}}=N n_{\mathrm{t}}$.

Applying the trough model the current-density equation becomes

$j=-q n_{\mathrm{d}} v_{\mathrm{d}}-q n_{\mathrm{s}} v_{\mathrm{s}}+q D_{n} \partial n / \partial x+\partial D / \partial t$

with $j$ the total current density, $-q$ the electron charge, $v_{\mathrm{d}}$ the drift velocity, $D_{n}$ the diffusion constant, $n$ the total electron density in the conduction band and $D$ the electric displacement.

Eqs. (1)-(3) are completed by Gauss' equation, the carrier-continuity equation, the piezo-electric relations and the wave equation. Note that the introduction of the trough model removes the need for cumbersome nonlinear equations. When deviations around a steady state are small the equations can be linearized.

For small deviations around the steady state we have in first order approximation

$\Delta p-\Delta b=-\Delta n_{\mathrm{t}} / \tau+(\partial p / \partial E-\partial b / \partial E)_{\Delta E=0} \Delta E$,

with

$\tau=-\left(\partial p / \partial n_{\mathrm{t}}-\partial b / \partial n_{\mathrm{t}}\right)_{\Delta n_{\mathrm{t}}=0}^{-1}$

the mean lifetime of fluctuations in the trough density. We assume in addition that the number of electrons per trough $N$ depends on the electric field strength; thence

$\Delta N=(\partial N / \partial E)_{\Delta E=0} \Delta E$.

Finally we obtain a set of 10 linear homogeneous differential equations in 10 variables, which can be solved by considering solutions of the type exp $i(\omega t-k x)$.

Solving for $k$ and using boundary conditions in accordance with experimental circumstances, for instance free end surfaces yielding zero stress at the boundaries, we obtain the following approximate expression for the ac small signal impedance $\neq 1$

$Z(\omega)=\frac{L}{A\left[\alpha /\left(\tau^{-1}+\mathrm{i} \omega\right)+\sigma_{1}+\sigma_{2}+\sigma_{3}+\mathrm{i} \omega \epsilon\right]}\left\{1-K_{\mathrm{e}}^{2} \frac{\left[\exp \left(-\mathrm{i} k_{1} L\right)-1\right]\left[\exp \left(-\mathrm{i} k_{2} L\right)-1\right]\left(\mathrm{i} k_{2} \beta_{1}-\mathrm{i} k_{1} \beta_{2}\right)}{k_{1} k_{2} L\left[\exp \left(-\mathrm{i} k_{1} L\right)-\exp \left(-\mathrm{i} k_{2} L\right)\right]}\right\}$,

where we used the reasonable assumption

$\left|K_{\mathrm{e}}^{2} \beta_{1,2}\right| \ll 1$,

with $L$ the contact spacing, $A$ the contact area, $K_{\mathrm{e}}=\left(e^{2} / \epsilon c\right)^{1 / 2}$ the electromechanical coupling factor, where $e$ is the piezo-electric constant, $\epsilon$ is the dielectric permittivity, $c$ is the elastic constant;

¥1 Details to be published elsewhere. 
$\alpha=q\left(\bar{v}_{\mathrm{d}}-v_{\mathrm{s}}\right) \bar{N}(\partial p / \partial E-\partial b / \partial E)_{\Delta E=0}$,

$\sigma_{1}=q \mu \bar{n}_{\mathrm{d}}$ with $\mu$ the electron mobility, $\sigma_{2}=q \mu^{\prime} \bar{n}_{\mathrm{s}}$ with $\mu^{\prime}$ the mobility of trapped electrons,

$\sigma_{3}=q\left(\bar{v}_{\mathrm{d}}-v_{\mathrm{s}}\right) \bar{n}_{\mathrm{t}}(\partial N / \partial E)_{\Delta E=0}, \quad k_{1}=\left(\omega / v_{\mathrm{s}}\right)\left(1-\frac{1}{2} K_{\mathrm{e}}^{2} \beta_{1}\right), \quad k_{2}=-\left(\omega / v_{\mathrm{s}}\right)\left(1-\frac{1}{2} K_{\mathrm{e}}^{2} \beta_{2}\right)$,

$\beta_{1}=\frac{1-\bar{v}_{\mathrm{d}} / v_{\mathrm{s}}-\mathrm{i} \omega / \omega_{\mathrm{D}}}{1-\bar{v}_{\mathrm{d}} / v_{\mathrm{s}}-\mathrm{i}\left[\omega / \omega_{\mathrm{D}}+\left(\alpha \tau+\sigma_{1}+\sigma_{2}+\sigma_{3}\right) / \epsilon \omega\right]}$,

$\beta_{2}=\frac{1+\bar{v}_{\mathrm{d}} / v_{\mathrm{s}}-\mathrm{i} \omega / \omega_{\mathrm{B}}}{1+\bar{v}_{\mathrm{d}} / v_{\mathrm{s}}-\mathrm{i}\left\{\omega / \omega_{\mathrm{D}}+\left[\alpha\left(\tau^{-1}+2 \mathrm{i} \omega\right)^{-1}+\sigma_{1}+\sigma_{2}+\sigma_{3}\right] / \epsilon \omega\right\}}$,

where $\omega_{\mathrm{D}}=v_{\mathrm{s}}^{2} / D_{\mathrm{n}}$ is the diffusion frequency.

Substituting reasonable values for the unknowns into eq. (6) it is found that the second term in eq. (6) only contributes significantly at frequencies given by

$f=(2 m+1) v_{s} / 2 L, \quad m=0,1,2,3, \ldots$.

Note that $L / v_{\mathrm{s}}$ is the transit time of sound waves.

Three limiting cases are of interest for our measurements:

(i) $\omega \rightarrow 0$, then (6) becomes

$Z(0)=L / A\left(\alpha \tau+\sigma_{1}+\sigma_{3}\right)$

( $\sigma_{2}$ vanishes, because $\mu^{\prime}$ is assumed to go to zero when $\omega \rightarrow 0$ );

(ii)

$\omega \gg\left|\sigma_{1}+\sigma_{2}+\sigma_{3}+\left[\alpha /\left(\tau^{-1}+i \omega\right)\right]\right| / \epsilon$,

then

$Z(\omega) \approx L / \mathrm{i} A \omega \epsilon$,

corresponding to the dielectric roll-off of a device with capacity $C=\epsilon A / L$;

(iii) intermediate frequencies, where

$\sigma_{1}+\sigma_{2}+\sigma_{3} \gg\left|i \omega \epsilon+\alpha /\left(\tau^{-1}+\mathrm{i} \omega\right)\right|$.

Then we find an impedance which is given by

$Z(\omega)=L / A\left(\sigma_{1}+\sigma_{2}+\sigma_{3}\right)$.

3. Experimental results and discussion. Measurements were carried out on semiconducting $\mathrm{CdS}(\rho=30 \Omega \mathrm{cm})$ with the electric field applied along the c-axis (typical dimensions $A=1 \times 1 \mathrm{~mm}^{2}, L=1.5 \mathrm{~mm}$ ). From the threshold voltage defined by the onset of the current fluctuations [8], Brillouin scattering data and the knee-voltage in the current-voltage characteristic it was clear that transversal off-axis waves were amplified in accordance with earlier reports [9].

Since off-axis waves with wave vectors making an angle from $10^{\circ}$ to $50^{\circ}$ with the direction of the electric field are expected to be amplified [10], the-resonances predicted by eq. (8) will be smoothed out over a larger frequency interval. This smoothing out will occur because in practice we have a distribution of trough velocities, which corresponds with a distribution of transit times.

Fig. 1 shows the result of an impedance measurement at $150 \mathrm{~V}$, a value which is $25 \mathrm{~V}$ above the treshold voltage defined by the onset of the current fluctuations [8]. Resonances appear at frequencies given by the odd harmonics 


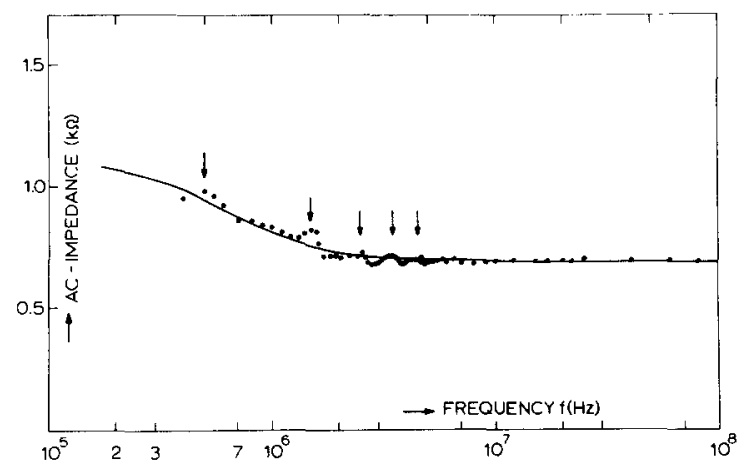

Fig. 1. Ac impedance of semiconducting CdS at $150 \mathrm{~V}(25 \mathrm{~V}$ above the threshold voltage). The solid line gives the best fit calculated impedance with the help of eq. (6). Resonance frequencies are indicated by arrows.

of $500 \mathrm{kHz}$. So from eq. (8) we find the trough velocity $v_{\mathrm{s}}=1.53 \times 10^{3} \mathrm{~m} \mathrm{~s}^{-1}$. The value of the trough velocity $v_{\mathrm{s}}$ is somewhat lower than the sound velocity of transverse waves $\left(1.8 \times 10^{3} \mathrm{~m} \mathrm{~s}^{-1}\right)$, but can be interpreted as the projection of the sound velocity of $30^{\circ}$-off-axis transverse waves on the direction of the electric field strength. Physically this would mean that troughs are carried along in the direction of the phase velocity of the amplified acoustic waves.

Using the threshold voltage $V_{\mathrm{c}}=125 \mathrm{~V}$ and the sound velocity $v_{\mathrm{s}}=1.53 \times 10^{3} \mathrm{~m} \mathrm{~s}^{-1}$ we find a mobility of $\mu$ $=v_{\mathrm{s}} L / V_{\mathrm{c}}=2 \times 10^{-2} \mathrm{~m}^{2} \mathrm{~V}^{-1} \mathrm{~s}^{-1}$, which is in accordance with mobility values in the literature [11]. The solid line in fig. 1 is calculated with the help of eq. (6). An unambiguous fit to the measurements can be obtained, if $\tau=1.79$ $\times 10^{-7} \mathrm{~s}, \alpha=-5.77 \times 10^{6}(\Omega \mathrm{m} \mathrm{s})^{-1}$ and $\sigma_{1}+\sigma_{2}+\sigma_{3}=2.77(\Omega \mathrm{m})^{-1}$. It should be noted that in eq. (6) we used $\mu^{\prime}=$ constant in the frequency range considered.

Additionally from the current-voltage characteristic we find at $150 \mathrm{~V}, \bar{n}_{\mathrm{d}}=6.1 \times 10^{20} \mathrm{~m}^{-3}$, so $\sigma_{1}=1.95(\Omega \mathrm{m})^{-1}$ and $\bar{n}_{\mathrm{s}}=4.3 \times 10^{20} \mathrm{~m}^{-3}$, and with the help of eq. $(10) \sigma_{3}=0.21(\Omega \mathrm{m})^{-1}$. It follows therefore that $\sigma_{2}=0.61$ $(\Omega \mathrm{m})^{-1}$ which means that $\mu^{\prime}=8.9 \times 10^{-3} \mathrm{~m}^{2} \mathrm{~V}^{-1} \mathrm{~s}^{-1}$.

From the above we can conclude that trapped electrons are less able to respond to an alternating external electric field than free electrons.

A value of $\alpha<0$ has the physical meaning that an increase in the applied voltage causes an increase in the number of troughs. On the other hand, the number of electrons per trough decreases when the voltage is increased, because $\sigma_{3}$ appears to be positive.

More experimental data are necessary to complete the physical picture regarding the voltage dependence of the introduced variables. However, it is clear that problems concerning the ac small signal impedance can be solved in a very comprehensible and simple way.

The authors are grateful to Professor Dr. C.Th.J. Alkemade for his critical reading of the manuscript. This work was performed as part of the research programme of the "Stichting voor Fundamenteel Onderzoek der Materie" (FOM) with financial support from the "Nederlandse Organisatie voor Zuiver Wetenschappelijk Onderzoek" (ZWO).

[1] D.L. White, J. Appl. Phys. 33 (1962) 2547.

[2] C.A.A.J. Greebe, Philips Res. Rep. 20 (1965) 1.

[3] G. Johri and H.N. Spector, Phys. Rev. B15 (1977) 4955.

[4] B. Shapiro, Phys. Rev. B12 (1975) 2402.

[5] P.K. Tien, Phys. Rev. 171 (1968) 970.

[6] A.R. Moore, J. Appl. Phys. 38 (1967) 2327.

[7] R.J.J. Zijlstra and P.A. Gielen, Physica 95B (1978) 190.

[8] P.A. Gielen and R.J.J. Zijlstra, Physica 95B (1978) 347.

[9] J. Zucker and S. Zemon, Appl. Phys. Lett. 9 (1966) 398.

[10] A.R. Moore, R.W. Smith and P. Worcester, IBM J. Res. Dev. (1969) 503.

[11] W.W. Spear and J. Mort, Proc. Phys. Soc. 81 (1963) 130. 